\title{
The Relationship of Amniotic Membrane 11-Oxidoreductase Activity to Lung Maturation in the Human Fetus
}

\author{
A. KEITH TANSWELL ${ }^{(30)}$ AND BARRY $\Upsilon$. SMITH \\ Department of Paediatrics, Queen's University, and the Neonatal Intensive Care Unit, Kingston General Hospital, \\ Kingston, Ontario, Canada
}

\section{Summary}

This study demonstrates that the ability of amniotic membranes to interconvert cortisone and cortisol is significantly different in samples from pregnancies in which the infant develops respiratory distress syndrome (RDS) when compared with those from normal unaffected infants. We first calculated the percentage of conversion of cortisone to cortisol. The values were plotted according to gestational age, the control group and RDS group identified separately, and regression lines calculated. Comparison of the two regression lines showed them to be significantly different $(P<$ 0.001). Comparison of expected values for all samples at a gestational age of $\mathbf{2 4 0}$ days again showed a highly significant difference $(P<\mathbf{0 . 0 0 1})$. After calculation of percentage of conversion of cortisol to cortisone, the regression lines for the two groups were shown to be not significantly different. However, after adjustment of data to a gestation period of $\mathbf{2 4 0}$ days the control group and the RDS group did show a difference $(P<0.01)$. For the "C-11 activation index," a measure of the net gain or loss of biological activity at the $\mathrm{C}-11$ position of the steroid molecule, direct comparison of regression lines failed to show a significant difference, but adjustment to $\mathbf{2 4 0}$ days of gestation showed the most significant difference seen between the two groups $(P<0.001)$. We also observed that when labor commences with spontaneous membrane rupture there is a significantly greater net production of cortisol by the membranes than if labor begins with contraction or bleeding $(P<0.001)$.

The fetus, by extracting cortisol from the amniotic fluid, can maintain a significant part of his circulating plasma cortisol from sources other than maternal blood or the fetal adrenal. Our findings are compatible with the concept that the amniotic membranes are a major source of the free cortisol found in amniotic fluid. Although it seems that the membranes act as an extraadrenal source of cortisol for the fetus, they probably share a control mechanism with the fetal adrenal.

\section{Speculation}

The appreciation of an extraadrenal source of glucocorticoids for the fetus may be only one example of a nonplacental origin for active compounds reaching the fetus, which may in the future be amenable for utilization or manipulation to the advantage of the preterm infant in utero.

We have previously demonstrated that interconversion of inactive cortisone (E) to active cortisol $(\mathrm{F})$ by human amniotic membrane results in significant net gain of glucocorticoid activity beyond the 31 st week of gestation, and rises with advancing gestational age (25). In the present study, this activity has been compared in infants with RDS, to control infants with normal postnatal lung function.

\section{MATERIALS AND METHODS}

Unlabeled cortisol (compound $\mathrm{F}$, or 4-pregnen-11 $\beta, 17 \alpha, 21$ triol-3,20-dione) and cortisone (compound $\mathrm{E}$, or 4-pregnen$17 \alpha, 21$-diol-3,11,20,-trione) were purchased from Steraloids Inc., Pawling, NY, and labeled steroids $\left[4-{ }^{14} \mathrm{C}\right]$ cortisone (spec act 59.8 $\mathrm{mCi} / \mathrm{mmol}$ ) and $\left[1,2-{ }^{3} \mathrm{H}\right]$ cortisol (spec act $40 \mathrm{Ci} / \mathrm{mmol}$ ) were purchased from New England Nuclear, Dorval, Quebec. All organic solvents were redistilled in glass. The method for measuring steroid interconversion has been described in detail previously (25). Washed and blotted samples of amniotic membrane weighing $100 \pm 5 \mathrm{mg}$ were mined finely with scissors and incubated for $2 \mathrm{hr}$ in $2 \mathrm{ml}$ Earle's balance salt solution under $5 \% \mathrm{CO}_{2}$ in air. The medium contained equimolar amounts $\left(8.3 \times 10^{-7} \mathrm{M}\right)$ of $\left[{ }^{14} \mathrm{C}\right]$ cortisone $\left(1.2 \times 10^{5} \mathrm{dpm}\right)$ and $\left[{ }^{3} \mathrm{H}\right]$ cortisol $\left(1.1 \times 10^{6} \mathrm{dpm}\right)$. After incubation the samples were frozen at $-20^{\circ}$ until analysis. Steroids were extracted with $5 \mathrm{vol}$ ethyl acetate containing $20 \mu \mathrm{g}$ unlabeled $E$ and $F$, and steroid separation achieved by thin layer chromatography on silica gel $\mathrm{G}$ in chloroform-methanol (9:1). Counting was performed in a Searle Isocap 300 liquid scintillation spectrophotometer. From the content of ${ }^{14} \mathrm{C}$ and ${ }^{3} \mathrm{H}$ in both $\mathrm{E}$ and $\mathrm{F}$ we were able to calculate 1) percentage of conversion of $E$ to $F, 2$ ) percentage of conversion of $F$ to $E$, and 3 ) by subtracting 2) from 1) we obtained the "C-11 activation index," a measure of the net gain or loss of biologic activity at the $\mathrm{C}-11$ position of the steroid molecule (19).

Clinical data was obtained by retrospective analysis of the maternal and neonatal medical records. Specific note was taken of maternal diabetes, maternal steroid therapy, delivery by cesarean section, intrauterine growth retardation (IUGR), prolonged rupture of membranes (i.e. $>24 \mathrm{hr}$ ), labor onsetting with membrane rupture, and RDS. Gestational age was assessed by menstrual data, and confirmed by the gestational assessment technique of Dubowitz et al. (7). A diagnosis of IUGR was made if the birthweight was below the 10th percentile for gestational age according to the data of Gairdner and Pearson (8). We made a diagnosis of RDS according to our own modification (24) of the criteria of Baden et al. (2).

All values were reported as means $\pm \mathrm{SD}$. The relationship of observed values to gestational age was compared by computing linear regression lines by the method of least squares for both the control and RDS populations. Differences between the two populations were compared by two methods, a) by comparing the slopes of each population by Student's $t$-test using the formula

$$
t=\frac{\mathrm{m}_{1}-\mathrm{m}_{2}}{\frac{\left(\mathrm{n}_{1}-1\right) \mathrm{SE}_{1}+\left(\mathrm{n}_{2}-1\right) \mathrm{SE}_{2}}{\left(\mathrm{n}_{1}-1\right)+\left(\mathrm{n}_{2}-2\right)}}
$$

where $t=$ Student's $t$ statistic, $\mathrm{m}_{1}=$ slope of control population, $\mathrm{m}_{2}=$ slope of RDS population, $\mathrm{SE}_{1}=\mathrm{SE}$ of the slope of the control population, $\mathrm{SE}_{2}=\mathrm{SE}$ of the slope of the RDS population, 
$\mathrm{n}_{1}=$ number of observations in the control population, $\mathrm{n}_{2}=$ number of observations in the RDS population, or b) by calculating expected values for each individual observation extrapolated to an arbitrary gestational age of 240 days, and comparing the populations using Student's $t$-test. The expected value at 240 days was calculated by the formula, $\mathrm{x}=\mathrm{m}(240-\mathrm{g})+\mathrm{y}$, where $\mathrm{x}=$ expected value at 240 days, $y=$ observed value, and $m=$ slope of the population from which y was derived.

\section{RESULTS}

The ability of all samples to convert $\mathrm{E}$ to $\mathrm{F}$, plotted according to gestational age, is shown in Figure 1. The control group and the RDS group are identified separately, and the regression line for each is shown (control group: $n=53$, slope $=0.5728$; RDS group $n=13$, slope $=0.0156$ ). Statistical comparison of the two regression lines shows them to be significantly different $(t=5.41, P$ $<0.001$ ). Regression lines for the conversion of $F$ to $E$ by both groups were calculated (control group: $n=53$, slope $=0.0910$; RDS group: $n=13$, slope $=0.1448$ ), and were not significantly different $(t=0.17, P=\mathrm{N}$. S.). Results and regression lines for the "C-11 activation index" of both groups are shown in Figure 2 (control group: $n=53$, slope $=0.4813$; RDS group: $n=13$, slope $=0.1604)$. The technique of regression line comparison fails to demonstrate the difference between these two groups $(t=0.69, P$ $=\mathrm{NS}$ ). Once all data had been adjusted to give expected values for a gestation period of 240 days, as described in Materials and Methods, it was possible to compare the two groups directly,

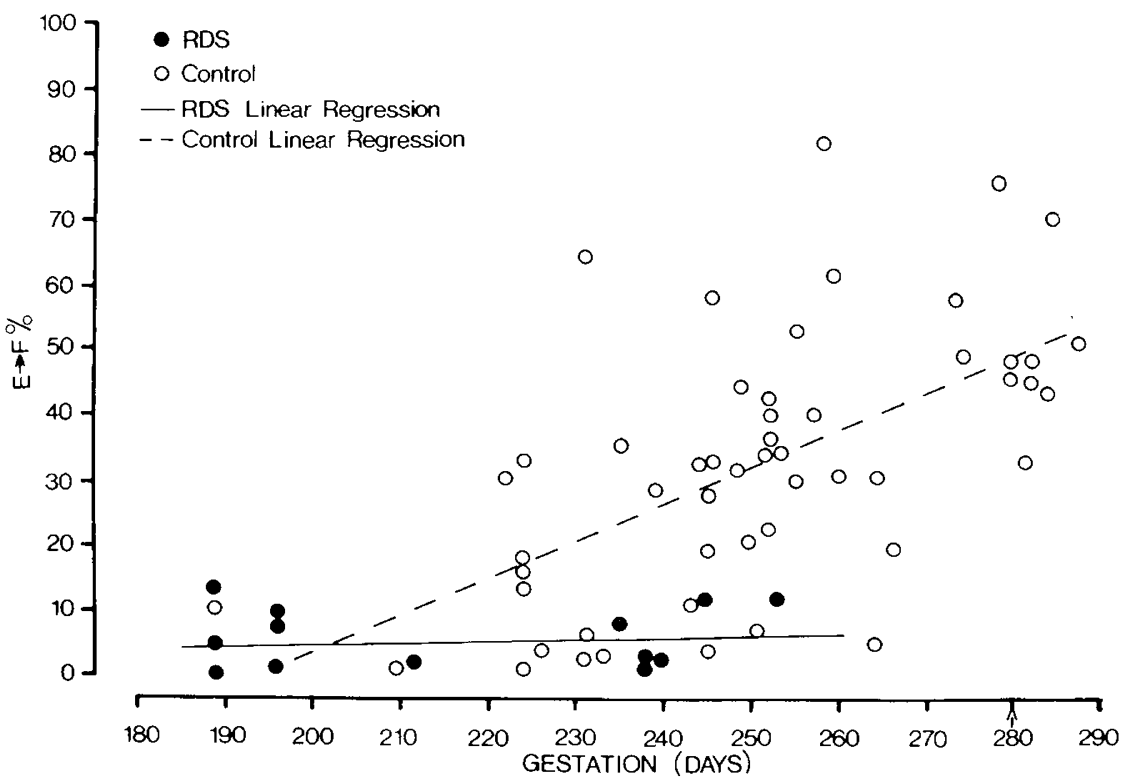

Fig. 1. Conversion (percentage) of $\left[{ }^{14} \mathrm{C}\right]$ cortisone (E) to cortisol (F) by human amniotic membrane plotted against gestational age at the time of delivery of control infants $(\bigcirc)$ and infants who developed RDS $(\bullet)$, with linear regression lines for both populations. Full term ( $\uparrow)$ is 280 days.

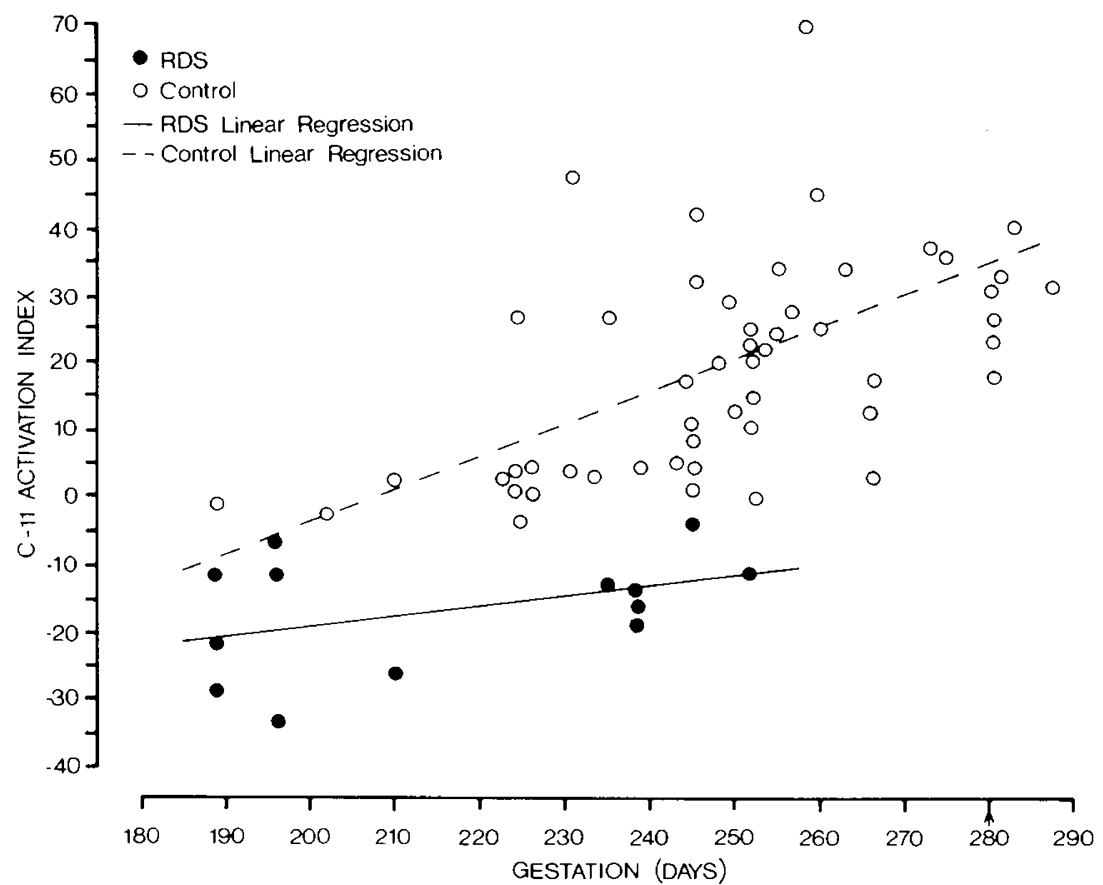

Fig. 2. The net gain of the active glucocorticoid, cortisol, resulting from the bidirectional interconversion of cortisone and cortisol (C-11 activation index, see Materials and Methods), plotted against gestational age at the time of delivery of control infants $(O)$ and infants that developed RDS $(\bullet)$, with linear regression lines for both populations. Full term ( $\uparrow)$ is 280 days. 
uninfluenced by gestation-dependent changes. The results are shown in Figure 3. Conversion of $\mathrm{E}$ to $\mathrm{F}$ (control group: $27.0 \pm$ 16\%; RDS group: $6.93 \pm 4.6 \% ; t=4.29$ ) and the C-11 activation index (control group: $15.4 \pm 14.3$; RDS group: $-12.5 \pm 7.8 ; t=$ 6.79) both showed highly significant differences $(P<0.001)$ between the two groups, being most apparent in the $\mathrm{C}-11$ activation index. Comparison of the two groups ability to convert $F$ to E (control group: $11.2 \pm 8.1 \%$; RDS group: $19.42 \pm 7.4 \% ; t=$ 3.37) showed a less significant difference $(P<0.01)$.

Of the 36 samples obtained between 230 and 260 days of gestation, 10 were associated with delivery by cesarean section, 2 with maternal diabetes, 6 with IUGR, and 2 with maternal steroid therapy. None of these results deviated significantly from the mean for the C-11 activation index of the control group.

This laboratory has previously demonstrated that if labor onsets with membrane rupture the infant is at reduced risk from RDS (26). We examined the effect of membrane rupture on the C-11 activation index. There were 17 samples with values $<0$, i.e. a net loss of biologic activity, of which 2 commenced labor with membrane rupture, compared with 17 of the 49 with values $>0$, i.e. a net gain in biologic activity. The difference was highly significant when the two groups were compared by chi-square analysis $\left(\chi^{2}\right.$ $=53.15, P<0.001)$. Thus when labor commenced with spontaneous membrane rupture there was a significantly greater production of cortisol by the membranes than if labor began with bleeding or contractions.

\section{DISCUSSION}

The present studies show that the ability of amniotic membrane to interconvert cortisone and cortisol is significantly different in samples from pregnancies where the infant develops RDS when compared with those from normal nonaffected infants, this difference being most apparent when the net activation of cortisol (C11 activation index) is calculated. The increase in conversion of cortisone to cortisol normally seen with gestation fails to occur in the RDS samples. In addition, labor commencing with membrane rupture (which may be associated (26) with a decreased incidence of RDS) were significantly more likely to be associated with net gain of biologic activity, i.e., a positive $\mathrm{C}-11$ activation index, than those commencing with contractions or bleeding.

A therapeutic role for enhancement of fetal lung maturation by exogenous steroids administered through the maternal circulation is well established $(4,10)$. Although a role for normal endogenous fetal glucocorticoids in lung maturation awaits final proof, it is probable that their increase in concentration with gestation influences pulmonary maturation in the same fashion $(3,22)$. Fetal plasma levels of the active glucocorticoid, cortisol, increase with gestational age (11) and are at lower levels in those infants that subsequently develop RDS (13). Similarly, a rise in cortisol levels in amniotic fluid occurs with gestation (14) and correlates well with fetal plasma levels but not with maternal plasma levels. Both the cortisol to cortisone ratio (21) and the cortisol level of amniotic fluid (9) are related to pulmonary maturation. We have previously calculated that a preterm infant is capable of supplying a major portion of his cortisol requirements from the amniotic fluid (25), and this concept is supported by recent observations in mammalian species of very rapid appearance in fetal plasma of intraamniotically injected radioactive cortisol (5). In 1969 Abramovich and Wade (1) observed that the majority of free cortisol found in amniotic fluid does not enter from the fetus, and that the free unconjugated fraction increases with gestation. There is only limited capacity for cortisol to be bound in amniotic fluid (6).

Since the increasing levels of free cortisol seen in amniotic fluid do not appear to derive from fetal urine or from maternal placental blood (12), its origin is obscure. The observation by Murphy (15) that amniotic membrane is capable of converting cortisone to cortisol at both late and midgestation suggests one possible source. We subsequently demonstrated that the net production of cortisol by the amniotic membrane first occurs at about 31 weeks of gestation and increases progressively to term (25). The low molec-

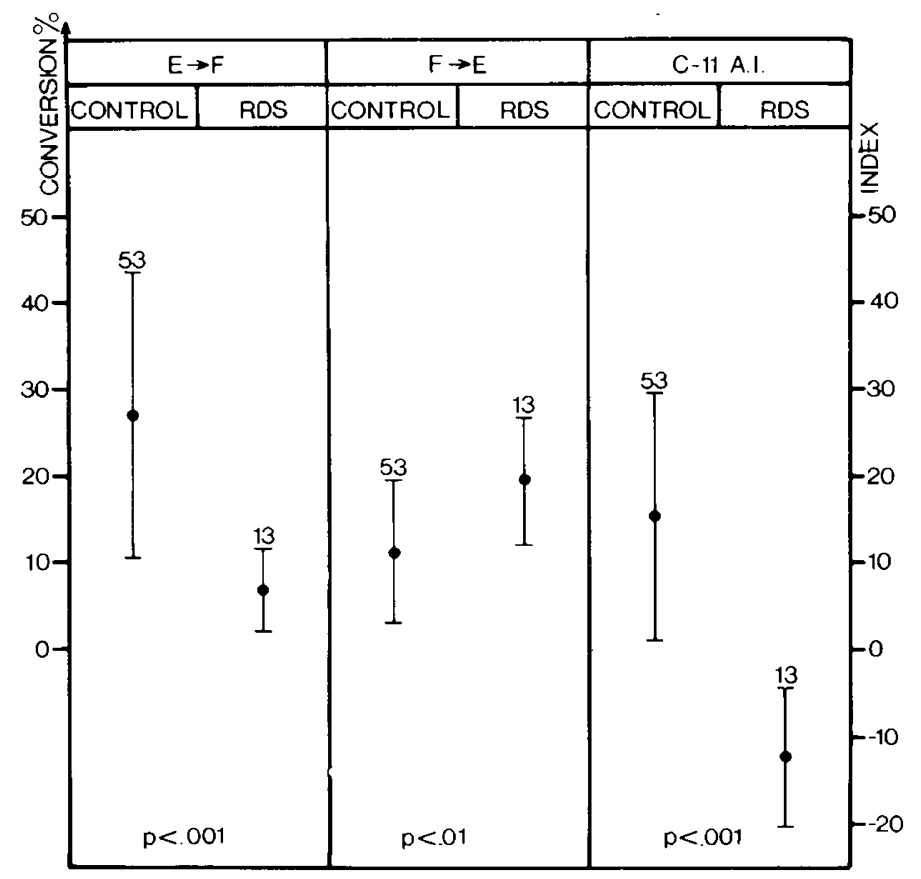

Fig. 3. Conversion (percentage) of ${ }^{14} \mathrm{C}$ cortisone (E) to cortisol (F), conversion (percentage) of $\left[{ }^{3} \mathrm{H}\right]$ cortisol (F) to cortisone (E), and net gain of cortisol (C-11 activation index) in human amniotic membrane from control infants and infants that developed RDS, the normal gestational changes having been eliminated by adjustment of data to an arbitrary uniform gestation of 240 days (see Materials and Methods).

ular weight and high lipid solubility of cortisol favors its diffusion across the amniotic membrane $(16,18)$.

The fetus, by rapidly and efficiently extracting cortisol from the amniotic fluid, can provide a significant portion of the cortisol found in fetal blood, in this fashion. The loss of this source of active glucocorticoid probably accounts for the fall in fetal plasma cortisol levels seen normally in the first few days following delivery (23). The amniotic fluid cortisol could enter the fetus either by absorption from swallowed amniotic fluid, although recent data (5) suggests that this cortisol is mostly conjugated and thus biologically unavailable, or by passive diffusion through the amniotic membrane into the fetal aspect of the placental circulation and thence to the fetus.

Our findings are compatible with the concept that the amniotic membranes are a major source of the free cortisol found in amniotic fluid. This is probably both actively derived, as we have postulated, but may also be inactively derived from the nonvillous uterus which is capable of increased $E$ to $F$ conversion in pregnancy (17). In situations of fetal adrenal insufficiency an exogenous source of cortisol would be most useful if under control different than that for the fetal adrenal. This does not seem to be the case with the amniotic membranes since cortisol levels in amniotic fluid from pregnancies where the fetus is anencephalic are low (20).

Thus, although it seems that the membranes act as an extraadrenal source of cortisol for the fetus, they probably share a control mechanism with the fetal adrenal.

\section{CONCLUSION}

Amniotic membrane samples from pregnancies in which the infant develops RDS are defective in their ability to interconvert cortisone and cortisol, and do not show the increasing ability to produce cortisol normally seen with advancing gestation. This may result in reduced levels of cortisol in both amniotic fluid and fetal blood, and thus affect fetal lung maturation adversely. At least one factor which may reduce the likelihood of RDS, labor 
onset with membrane rupture, is associated with enhanced cortisol production by the amniotic membranes.

\section{REFERENCES AND NOTES}

1. Abramovich, D. R., and Wade, A. P.: Transplacental passage of steroids: The presence of corticosteroids in amniotic fluid. J. Obstet. Gynaecol. Br. Commonw., 76: 610 (1969).

2. Baden, M., Bauer, C. R., Colle, E., Klein, G., Taeuch, H. W., and Stern, L.: A controlled trial of hydrocortisone therapy in infants with respiratory distress syndrome. Pediatrics, 50: 526 (1972).

3. Ballard, P. L., Benson, B. J., and Brehier, A.: Glucocorticoid effects in the fetal lung. Amer. Rev. Resp. Dis., 115(ii): 29 (1977).

4. Ballard, R. A., Sniderman, S., Ballard, P. L., and Granberg, P.: Prenatal betamethasone for the prevention of ideopathic respiratory distress syndrome [Abstr.] Clin. Res., 25: 187A (1977).

5. Carson, G. D., and Challis, J. R. G.: Personal communication

6. Challis, J. R. G., and Bennett, M. J.: Cortisol binding in human amniotic fluid. Amer. J. Obstet. Gynecol., In press.

7. Dubowitz; L. M. S., Dubowitz, V., and Goldberg, C.: Clinical assessment of gestational age in the newborn infant. J. Pediat., 77: 1 (1970).

8. Gairdner, D., and Pearson, J.: A growth chart for premature and other infants. Arch. Dis: Childhood, 46: 783 (1971).

9. Gewolb, I. H., Hobbins, J. C., and Tan, S. Y.: Amniotic fluid cortisol as an index of fetal lung maturity. Obstet. Gynecol., 49: 462 (1977).

10. Liggins, G. C., and Howie, R. N.: A controlled trial of antepartum glucocorticoid treatment for prevention of the respiratory distress syndrome in premature infants. Pediatrics. 50: 515 (1972).

11. Murphy. B. E. P., and Diez d'Aux, R. C.: Steroid levels in the human fetus: Cortisol and cortisone. J. Clin. Endocrinol. Metab., 38: 158 (1974).

12. Murphy, B. E. P., Clark, S. J., Donald, I. R., Pinsky, M., and Vedady, D.: Conversion of maternal cortisol to cortisone during placental transfer to the human fetus. Amer. J. Obstet. Gynecol., 118: 538 (1974).

13. Murphy, B. E. P.: Evidence of cortisol deficiency at birth in infants with the respiratory distress syndrome. J. Clin. Endocrinol. Metab., 38: 158 (1975).

14. Murphy, B. E. P., Patrick, J., and Denton, R. L.: Cortisol in amniotic fluid during human gestation. J. Clin. Endocrinol. Metab., 40: 164 (1975).

15. Murphy, B. E. P.: Abstracts of the VIth International Congress of Endocrinology,
Hamburg, 1976, p. 108.

16. Murphy, B. E. P.: Chorionic membrane as an extra-adrenal source of fetal cortisol in human amniotic fluid. Nature, 266: 179 (1977).

17. Murphy, B. E. P.: Conversion of cortisol to cortisone by the human uterus and its reversal in pregnancy [Abstr.]. Clin. Res., 24: 661A (1977)

18. Seeds, A. E., Eichorst, B. C., and Stolee, A.: Factors determining human laeve permeability in vitro. Amer. J. Obstet. Gynecol., 128: 13 (1977).

19. Smith, B. T., and Giroud, C. J. P.: Effects of cortisol on serially propagated fibroblast cell cultures derived from the rabbit fetal lung and skin. Canad. J. Physiol. Pharmacol., 53: 1037 (1975).

20. Smith, B. T., and Worthington, D.: Discordant lung maturation and corticosteroid levels in twins. Pediat. Res., 10: 468 (1976).

21. Smith, B. T., Worthington, D., and Maloney, A. H. A.: Fetal lung maturation. III. The amniotic fluid cortisol/cortisone ratio in preterm human delivery and the risk of respiratory distress syndrome. Obstet. Gynecol., 49: 527 (1977).

22. Solomon, S.: Glucocorticoid binding: stimulus for lung development. Report of the Seventieth Ross Conference on Pediatric Research, Puerto Rico, 1975, p. 56.

23. Stevens, J. F.: Plasma cortisol levels in the neonatal period. Arch. Dis. Childhood, 45: $592(1970)$

24. Tanswell, A. K., Sherwin, E., and Smith, B. T.: The single step gastric aspirate shake test. Arch. Dis. Childhood, 52: 541 (1977)

25. Tanswell, A. K., Worthington, D., and Smith, B. T.: Human amniotic membrane corticosteroid 11-oxidoreductase activity. J. Clin. Endocrinol. Metab., 45: 721 (1977).

26. Worthington, D., Maloney, A. H. A., and Smith, B. T.: Fetal lung maturation. I. Mode of onset of premature labour: Influence of premature rupture of the membranes. Obstet. Gynecol., 49: 275 (1977).

27. This research was supported by a grant (MA 5757) from the Medical Research Council of Canada.

28. Dr. A. Keith Tanswell is a recipient of Fellowships from the Canadian Cystic Fibrosis Foundation and the William T. McEachern Foundation.

29. Dr. Barry T. Smith is a Scholar of the Medical Research Council of Canada

30. Requests for reprints should be addressed to: Dr. A. K. Tanswell, Department of Paediatric Research, Queen's University, 146 Stuart Street, Kingston, Ontario K7L 2V8 (Canada).

31. Received for publication August 22, 1977.

32. Accepted for publication December 14, 1977. 